\title{
Using Noninvasive Brain Measurement to Explore the Psychological Effects of Computer Malfunctions on Users during Human-Computer Interactions
}

\author{
Leanne M. Hirshfield, ${ }^{1}$ Philip Bobko, ${ }^{2}$ Alex Barelka, ${ }^{3}$ Stuart H. Hirshfield, ${ }^{4}$ \\ Mathew T. Farrington, ${ }^{4}$ Spencer Gulbronson, ${ }^{4}$ and Diane Paverman ${ }^{4}$ \\ ${ }^{1}$ M.I.N.D. Lab, Department of Communications, SI Newhouse School, Syracuse University, Syracuse, NY 13244, USA \\ ${ }^{2}$ Departments of Management and Psychology, Gettysburg College, Gettysburg, PA 17325, USA \\ ${ }^{3}$ Department of Management and Quantitative Methods, Illinois State University, Normal, IL 61761, USA \\ ${ }^{4}$ Department of Computer Science, Hamilton College, Clinton, NY 13323, USA
}

Correspondence should be addressed to Leanne M. Hirshfield; lmhirshf@syr.edu

Received 20 September 2013; Accepted 17 March 2014; Published 30 April 2014

Academic Editor: Francesca Morganti

Copyright ( 2014 Leanne M. Hirshfield et al. This is an open access article distributed under the Creative Commons Attribution License, which permits unrestricted use, distribution, and reproduction in any medium, provided the original work is properly cited.

In today's technologically driven world, there is a need to better understand the ways that common computer malfunctions affect computer users. These malfunctions may have measurable influences on computer user's cognitive, emotional, and behavioral responses. An experiment was conducted where participants conducted a series of web search tasks while wearing functional nearinfrared spectroscopy (fNIRS) and galvanic skin response sensors. Two computer malfunctions were introduced during the sessions which had the potential to influence correlates of user trust and suspicion. Surveys were given after each session to measure user's perceived emotional state, cognitive load, and perceived trust. Results suggest that fNIRS can be used to measure the different cognitive and emotional responses associated with computer malfunctions. These cognitive and emotional changes were correlated with users' self-report levels of suspicion and trust, and they in turn suggest future work that further explores the capability of fNIRS for the measurement of user experience during human-computer interactions.

\section{Introduction}

As the lines between humans and computers become blurred, research is beginning to measure users' experiences while interacting with a technology or information system. In particular, dealing with computer malfunctions that involve slow internet connectivity, or those that involve the introduction of malware onto one's computer system, has unfortunately become a somewhat regular part of users' interactions with computers. Computer users' cognitive load and emotional state may change depending on the type and severity of the malfunction. Additionally, one's perceived trust in, or suspicion of, the computer system may be correlated with these changes in cognitive load and emotional state.

It is commonplace to use surveys to acquire users' selfreports of their cognitive load and psychological states during human-computer interactions. For example, the NASA-TLX is one of the most commonly used surveys for assessing workload [1]. As another example, when attempting to measure self-report emotional states, users often complete surveys such as semantic differentials, the Self-Assessment Manikin, or the Positive and Negative Affect Schedule [2, 3]. The vast majority of trust research to date has also relied on surveys to assess people's trust in others $[4,5]$. Although this method of measurement is commonplace and valuable for understanding and measuring changes in user states, it is limited by many of the well-known drawbacks of subjective, self-report measures. For example, subjects may have different frames of reference when completing surveys; further, survey responses correlate only moderately with actual behavior and/or others' perceptions of the subject's behavior [6]. Also, subjects' use of rating scales is prone to 
distortion due to social desirability [7], and surveys and selfreports are often administered after a task has been completed (postdictively). They are thus limited in their capacity to accurately collect valuable insight into the users' changing experiences throughout a task.

To compensate for the shortcomings of subjective, selfreport techniques, in this study we use noninvasive brain measurement techniques to measure changes in user states objectively and in real time. Such measurement techniques have emerged in the literature with fMRI and the electroencephalograph (EEG) being used to measure workload and emotional states in human-computer interactions [814]. Furthermore, some researchers have recently used fMRI and associated brain activity to measure aspects of trust and distrust $[15,16]$. Although fMRI provides valuable information about cognitive functioning in the brain, the device is quite constricting. It requires subjects to lie still in a large magnet and is extremely expensive $[17,18]$. Although fMRI results suggest that we can measure trust objectively by assessing brain functioning, the tool cannot be used outside the research lab, limiting its uses for monitoring trust in more operational, real-world situations.

In order to enable the measurement of cognitive load, emotion, and the correlated constructs of trust and suspicion in real-world contexts, we employed a new, noninvasive brain sensing technique called functional near infrared spectroscopy (fNIRS) to make real-time, objective measurements of users' mental states while they conduct tasks in operational working conditions. The fNIRS device (shown in Figure 1) is easy to set up, lightweight, comfortable, and portable, and it can be implemented wirelessly, allowing for use in many settings.

One overarching goal in this study was to demonstrate the feasibility of using fNIRS to objectively measure users' states in real time while they work with, and interact with, computer systems. Towards these ends, we first provide a summary of the literatures on workload, emotional state, trust, and suspicion. We also describe specific research that guided our experimental goals and hypotheses [19]. We then describe our protocol, data analysis techniques, findings, and interpretations. We conclude with a discussion of the implications of this work for future research.

\section{Background and Literature Review}

2.1. Workload and Emotional State. The term cognitive workload is used in literature from various fields. Many describe cognitive workload in general terms, for example, as the ratio of the cognitive resources needed to complete a task to the cognitive resources available from the human operator [20]. Some view workload as a measure that can be determined subjectively, as done with the NASA TLX [1]. Others view workload via performance measurements, focusing on the operator's performance on a given task to determine levels of cognitive workload [20]. Yet others view cognitive workload as a measure of the overall activation measured by various brain imaging devices while subjects complete some task $[8,21,22]$. Cognitive psychologists note that there is not one

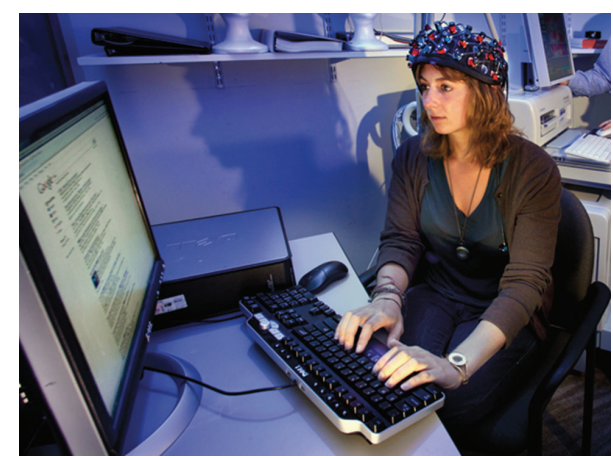

FIGURE 1: A subject wearing a 52-channel fNIRS device.

area in the brain that activates when a person is experiencing mental workload. However, these researchers look at specific areas in the brain to see which areas are activated while subjects perform simple tasks [23-25]. We have used fNIRS to measure spatial and verbal working memory load, as well as response inhibition load and visual search load [11, 12].

Regarding emotional reactions, most researchers agree that emotions are affective states that exist in a short period of time and are related to a particular event [26, 27]. From a psychological point of view, emotions are often mapped to points in a two-dimensional space of affective valence and arousal. Valence represents overall pleasantness of emotional experiences and arousal represents the intensity level of emotion, ranging from calm to excited [2, 28, 29]. These 2 dimensions enable researchers to differentiate among four categories of emotions. Some researchers even differentiate among nine categories of emotion by including a neutral section on both the valence and arousal axis. However, in principle, an infinite amount of other arbitrary numbers of categories can be defined [30].

\subsection{Trust and Suspicion}

2.2.1. The Agent of Trust and Suspicion. There is substantial literature exploring interpersonal trust-often in the management and other social science domains [4, 31-35] As the interactions between humans and their computer interfaces become increasingly "personal," research in the information technology (IT) realm is broadening this context to explore an individual's trust and distrust based on interactions not only with another person, but also with other types of external agents (e.g., computers and information systems). Across the literature on trust and automation [35-37], we note that the concept of trust has been (or can be) applied to trust in

(i) the operator of another IT system,

(ii) the programmer/designer of the IT system,

(iii) the programmer/developer of the algorithms used in the software,

(iv) the software itself (without reference to the programmer), and

(v) the hardware in the system. 
2.2.2. Trust (and Distrust). Rousseau et al's review on the topic of trust in the management domain concluded that there are many definitions of trust [31]. Mayer et al's definition of interpersonal trust is most frequently cited (including the three trustworthiness components of ability, integrity, and benevolence) [32]. Rousseau et al.s review leads to a similar definition of trust; that is, "a psychological state comprising the intention to accept vulnerability based upon positive expectations of the intentions or behavior of another" (page 395). This is consistent with Lyons et al.'s (2011) recent definition in IT contexts, and we adopt it here, with the modification that "another" can be "another person" or "another agent" such as an IT system [19].

2.2.3. Suspicion. The construct of suspicion seems related to trust and distrust, yet there is scant literature available on this topic; let alone literature that considers suspicion in IT contexts. Thus, we also investigate the concept of suspicion, but in an exploratory manner. Based on work in social psychology, marketing, communication, human factors, and management, Bobko et al. [38] define state suspicion as the "simultaneous combination of uncertainty, perceived (mal) intent, and cognitive activity (searching for alternative explanations or new data)." Note that a key component of suspicion is uncertainty-and it occurs with a negative frame ("concern") that is linked to cognitive activity regarding the uncertainty. In one of the few empirical papers in this area, Lyons et al. preliminarily investigated suspicion, trust, distrust, and an operator's decision confidence [19].They also stated that trust, distrust, and suspicion are orthogonal constructs, although their conclusions are potentially confounded by the referents used (e.g., suspicion was about the subject's particular computer, whereas trust and distrust were about an IT system). As noted above, most, if not all, measures of trust and distrust in the literature are self-report assessments that ask the trustor to respond using Likert scales or behavioral checklists. The scarce literature on suspicion uses the same methodology [38].

\subsection{Users' Cognitive and Emotional Reactions to Computer} Malfunctions. When users are confronted with unexpected stimuli during their human-computer interactions, we expect them to have a negative emotional response, and we expect a decrease in trust to be correlated with that emotional response. In some scenarios, the users may become frustrated with the computer ("This computer always freezes on me!"), or in other instances they may even become concerned that a malevolent agent has gained access to their computer ("I think this website stole my credit card information!"). Both examples imply that the computer user's beliefs about the cause of an unexpected stimulus will affect his or her physiological response to that stimulus; that is, characteristics of the unexpected stimulus moderate the physiological, cognitive, and emotional responses.

Regarding potential emotional responses, Figure 2 shows two variants of Russell's circumplex model of affect, arguably the most popular model depicting the arousal and valence dimensions and their relation to emotional state [28].
Research states that most people's neutral (a.k.a baseline) state is located near point $(0,0)$ in Russell's model [28]. The current study focuses primarily on the model to the left in Figure 2. We symbolically place our hypotheses on the model in the left panel of Figure 2, while the depiction in the right panel of Figure 2 contains more detailed semantic descriptors. Indeed, note that our first example above ("this computer always freezes on me") was suggested as inducing user frustration. In Figure 2, "frustration" is associated with high negative valence, but only moderate arousal (see Hla in Figure 2). In contrast, our second example above ("I think this website stole my credit card information") was suggested as inducing user reactions of concern, alarm, and fear. In Figure 2, these terms are associated with high arousal, but only moderate negative valence (posited in Figure 2 as H1b).

With these depictions in mind, we hypothesize the following.

H1a. If unexpected computer-generated stimuli are negative, but minor, they will cause reactions similar to being frustrated and/or annoyed. More specifically, when compared to a user's baseline state, unexpected minor negative stimuli will be associated with a moderate increase in arousal and a large decrease in valence, as indicated by the letter "H1a" in Figure 2.

H1b. If unexpected computer-generated stimuli are perceived as severely negative, or they are attributed to possible malintent by an external agent, they will cause individuals to feel afraid and/or alarmed. More specifically, when compared to a user's baseline state, the presence of these unexpected and severely negative stimuli will be associated with a large increase in arousal and only a moderate decrease in valence, as indicated by the letter "H1b" in Figure 2.

fMRI methods have recently been used to identify neural networks in the brain that are associated with different semantic emotional states [39] and with Russell's 2-dimensional valence/arousal model [40]. However, this research remains in the nascent stage, and more work is needed to understand the neural correlates of emotion. In particular, the the neural correlates of mental states such as fear or alarm (i.e., the regions associated with the "H1b" label in Figure 2) remain largely unexplored in the research literature. In the limited work that has been done with fMRI, the emotional state of "fear" has been found to activate areas in the dorsolateral prefrontal cortex (DLPFC), in the preand supplementary motor cortex, and in Broca's area [13]. Furthermore, research has found that high levels of stress and arousal have a direct effect on Broca's area [41]. Also, activation in the orbitofrontal cortex has been linked to an "alarm signal" that is sent by the brain in response to negative affect, when there is a need to regulate the negative emotion [42]. Lastly, much research has linked DLPFC activation to the cognitive load associated with regulating one's emotions such as frustration or fear (i.e., the regions labeled with the "H1a" and "H1b," respectively, in Figure 2) [13, 17].

Although all of the above brain measurement research was done with fMRI, fNIRS sensors, with a depth into the brain of up to $3 \mathrm{~cm}$, are capable of reaching Broca's area, the DLPFC, the supplementary motor cortex, and the 


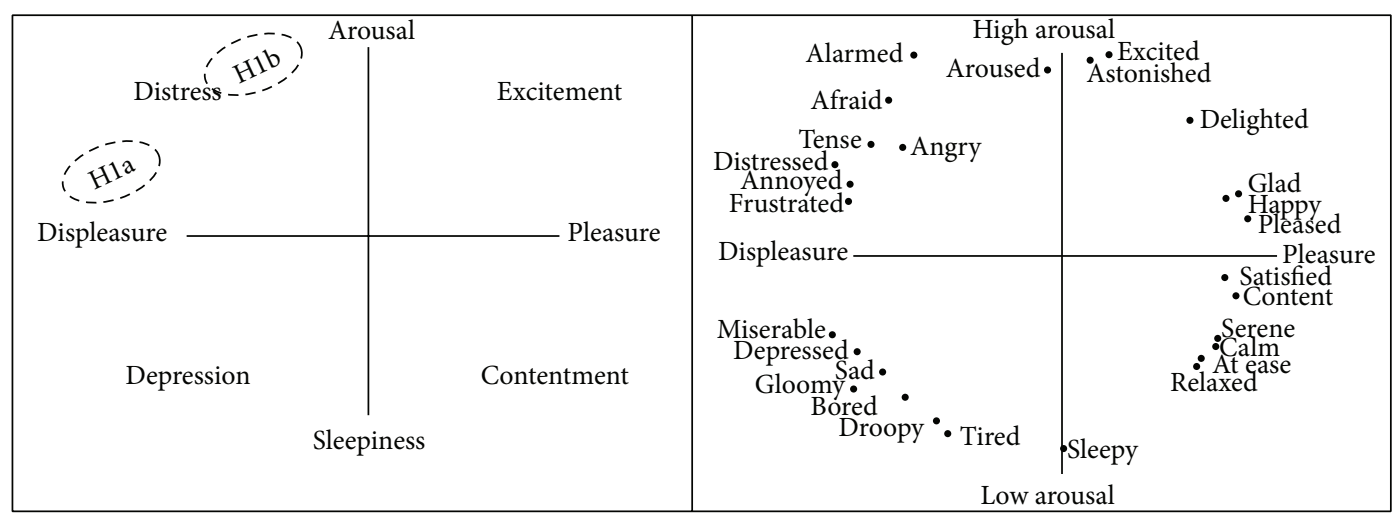

Figure 2: Two schematics of Russell's circumplex model of affect [28]. As we are less interested in semantic meaning than we are in the physiological responses related to our experimental stimuli, we focus on the model to the left.

orbitofrontal cortex. This suggests that the neural correlates relating to high arousal and moderately low levels of valence can be measured noninvasively. This leads us to an instrumentation corollary to our first set of hypotheses:

H1 Corollary. The regions identified by $\mathrm{Hla}$ and $\mathrm{H} 1 \mathrm{~b}$ in Figure 2 can be distinguished noninvasively, with fNIRS and GSR sensors.

In addition to affective state, we also expect computer malfunctions to affect users' cognitive load (and to reduce trust in a computer system). The overall cognitive load required to perform a task using a computer is composed of a portion attributable to the difficulty of the task itself plus a portion attributable to the complexity of operating the computer. In this regard, we follow Shneiderman's theory of syntactic and semantic components of a user interface [43]. The semantic component involves the workload needed to complete the task. The syntactic component includes interpreting the computer's feedback and then formulating and inputting commands to the computer. A goal in user interface design is to reduce the mental effort devoted to the syntactic aspects so that more workload can be devoted to the underlying task, or semantic, aspects [11]. People prefer to expend as little cognitive effort as possible when working with a computer system, and well-designed systems are able to minimize syntactic workload in order to meet this important user goal.

Therefore, when a computer malfunctions, we expect to see increases in the users' cognitive load and a potential loss of trust, as the user is forced to account for the shortcomings of the computer. For example, if the computer is performing slowly while a user tries to compose an email, the user may need to use more verbal working memory load while she keeps her train of thought and waits for the computer to catch up. Or if a user is working with a poorly designed software program, he may continually have difficulties finding the correct menu items and commands to achieve his desired outcome. Accompanying his loss of trust in the software will be an increase in cognitive load as he tries to navigate the interface and complete his target task. Literature has also shown that increases in negative affect are directly related to increases in cognitive load $[17,44]$. While this cognitive load can take many forms in the brain, one form involves activation in the DLPFC brain region, which has been linked to the cognitively demanding effort involved in emotion regulation [45].

This leads to our next hypothesis.

H2. Computer users will experience more cognitive load when interacting with a malfunctioning computer than they did when working on a properly functioning machine.

2.3.1. MRI Studies Related to Trust and Suspicion. The current experiment focuses on the changing cognitive and emotional state changes that result from two common computer malfunctions. A secondary experimental goal is to explore the way that users' levels of trust and/or suspicion may be related to these measured cognitive and emotional state changes. Several brain regions that are of interest in trust research relate to a paradigm called "theory of mind" (ToM; Premack and Woodruff, 1978 [46]), which is concerned with understanding how individuals attribute beliefs, desires, and intentions to oneself and others. Researchers conducting ToM studies have found that the anterior paracingulate cortex is activated when participants are deciding whether or not to trust someone else $[15,16]$. The anterior paracingulate cortex is a subset of the anterior cingulate cortex, which can be measured by fNIRS. Krueger et al. used fMRI to measure the brain activity of pairs of people playing a classic trust game [16]. They found that building a trust relationship was related to activation in the paracingulate cortex, which (as shown in the ToM research stated above) is involved in the process by which we infer another's intentions. They also found that unconditional trust was related to activity in the septal area, a region that has been linked to social attachment behavior. Dimoka constructed a study that mimics typical interactions with e-bay sellers. She asked participants, while in an MRI machine, to complete a series of purchasing interactions with hypothetical "sellers" [15]. She noted that participant's thoughts when working with "low distrust" sellers were associated with brain activation in the anterior paracingulate cortex. 
In summary, the fMRI research presented in this section suggests that higher brain activation in the anterior paracingulate cortex will be associated with the process by which users conduct the cognitively demanding process to infer whether or not another person is trustworthy (actively trying to infer another's intentions). Because people tend to be cognitive misers, they prefer to adopt truth (or lie) biases that enable them to unconditionally trust (or distrust) others and thereby reduce their cognitive load [47, 48]. Thus, we suggest that individuals tax the paracingulate cortex when there is a need to place cognitive effort toward the determination of an external agent's intentions. This may also indicate suspicion on the part of the user, because cognitive activation (generation of alternative possible explanations for observed behavior) is an important component of state suspicion [38]. This leads to our third hypothesis.

H3. Increases in paracingulate cortex activity will be associated with increased suspicion in individuals as they conduct the cognitively demanding process involved in inferring the intentions of others.

Research has also suggested that if an individual is uncertain about the intentions of another (or uncertain about any number of other interactions within their environment, and that uncertainty is perceived to have potentially negative consequences), then anxiety will increase $[14,38]$. Because uncertainty is a component of state suspicion, and subsequent anxiety is associated with increases in arousal (see Figure 2); we further hypothesize the following.

H3a. Suspicion will be accompanied by an increase in physiological indices of arousal.

2.3.2. The Utility of Functional Near-Infrared Spectroscopy. Users in the brain imaging studies described above were placed in cumbersome, expensive, and constricting fMRI scanners during all studies. There is a need to study trust, distrust, and suspicion, as well as their associated affective states, while computer users conduct more naturalistic humancomputer interactions. To this end, we use the noninvasive fNIRs and GSR sensors in our study.

The fNIRS device was introduced in the 1990's to complement, and in some cases overcome, the limitations of the EEG and other brain imaging techniques [49]. The fNIRS device uses light sources in the wavelength range $(690-830 \mathrm{~nm})$ that are pulsed into the brain. Deoxygenated hemoglobin $(\mathrm{Hb})$ and oxygenated hemoglobin ( $\mathrm{HbO}$ ) are the main absorbers of near-infrared light in tissues during hemodynamic and metabolic changes associated with neural activity in the brain [49]. These changes can be detected by measuring the diffusively reflected light that has probed the brain cortex $[21,49,50]$. We have used fNIRS to successfully measure a range of cognitive states [11, 12, 18, 51, 52] while computer users complete tasks under normal working conditions and, as noted above, one purpose of the current study is to further explicate the utility of fNIRS measurements.

2.4. Experimental Design. Eleven individuals (4 males) participated in this study (mean age $=20.2$ years; $S D=0.603$ ).
Participants were college students representing a range of majors. Informed consent was obtained, and participants were compensated for their time. All participants filled out a preexperimental survey, which included demographic information and computer usage queries. All participants answered that they frequently used the Internet and had previous experience shopping online. Our experimental protocol was designed to begin with the participant at a level of normalcy and trust and to end with the participant in a state of distrust.

2.5. Task and Manipulations. Participants were asked to use the Google search engine to shop online for the least expensive model of a specified bike. We chose this search engine task because it involved a task with which most subjects had previous experience (i.e., online shopping). During each session, subjects had fifteen minutes to search for three specific bicycles online. Participants received financial bonuses for finding bicycles priced below a given benchmark price. Financial bonuses were calculated as a percentage of the subject's discount on the benchmark price. Thus, participants had incentive to continue searching for the lowest possible price on all bikes during their entire 15 minute task session. The participants were specifically instructed to use only the Google search engine while searching for the bikes. The participants were told that the objective of the study was to measure workload levels as users navigate shopping websites.

Each participant completed this 15-minute-long task four times over the course of four consecutive days. Participants were told that there would be five such sessions, but as described below, our intervention on day four obviated the need for a fifth session. During each session, the participant was placed in a small room containing the fNIRS device and a standard desktop computer to use while shopping. Placing the participant in a room alone was intended to distance the subject from the researchers. This distance prevented the subject from relying on the researchers when manipulations occurred (see below). Also in the participant's room was a "Call Researchers Button" that would alert the researchers if assistance was required. Participants were told to use the button only if they felt that the experiment needed to be stopped or if they required researcher intervention (i.e., if they felt uncomfortable with the measurement devices, or if they wanted to end the study for some reason).

2.5.1. Days One and Two: Baseline. During the first two days, each participant conducted his/her 15-minute-long task without any intervention on the part of the researchers; that is, participants searched for the lowest price of three bikes that were assigned to them without any other intervention. The purposes of these two sessions were to (a) establish participant familiarity with both the computer system and with the researchers and (b) establish positive, consistent interactions during the computer tasks. We felt that these two aspects would create a sufficient level of trust that could be weakened by subsequent manipulations (and our manipulation check confirms this; see next section). 
2.5.2. Day Three: Slowed Internet Manipulation. This manipulation was created to target Hypothesis la (among others), where the unexpected manipulation was negative, but minor. During the third 15-minute-long session we let users work for several minutes on the search task before introducing any changes. Our manipulation followed a set of carefully scheduled variations of the speed of the Internet. Levels of Internet speed were based upon a previous pilot study. The levels were chosen because they were overt enough to cause a noticeable delay in the Internet speed, yet subtle enough to remain within a speed range that subjects considered to be a frustrating, but believable, speed. Thus, the manipulation on day three was intended to induce frustration and to lower users' trust in the computer system. The lowered trust was expected because the slowdown could create reductions in perceived ability/integrity of the system.

2.5.3. Day Four: Malware Manipulation. This manipulation was created to test Hypothesis $1 \mathrm{~b}$, where the stimuli are perceived as severely negative, or they are attributed to malintent. On day four, subjects again had to shop for three bicycles online. No manipulations were introduced while searching for the first two bikes (which took approximately 10 minutes out of the day four session). We did this to reestablish any trust that may have been lost during the day three Internet speed manipulation. However, on this fourth day, the third bicycle presented to subjects was fictitious, and it could only be found on our custom website, "XtremeBestPrice.com" (this website is no longer accessible online as we did not want people outside of our study to stumble upon the fake malware site). This website was Google-indexed, and we purposely made some common web page design flaws (e.g., flashing animations, a few misspelled words) on the page in order to lower its trustworthiness (cf. Lee and See [35]). There was no indication that the researchers were responsible for its existence. We also wrote about our website in several web forums in order to add legitimacy to the website. When participants made their way to our website, they did so using the same methods that they had previously used on many occasions. As the participant navigated around our site, a series of pop-ups and downloads were automatically triggered, eventually launching a "Blue Screen of Death" to indicate a computer crash (this process is depicted in Figure 3). There was no way to exit this blue screen, so participants had little option but to call the researchers into the room using the "Call Researchers Button".

2.5.4. Debriefing and Suspicion Exit Survey. At the end of the fourth day, we debriefed subjects about the true nature of our study. After explaining that we had indeed caused the Internet to slow down on the third day and that we had created the fake xtremebestprice.com website, we asked subjects (via an open-ended survey) whether or not they had suspected that we, the researchers, rather than the internet connection or the website, were the cause of the computer glitches.

2.6. Equipment Set-Up. We collected fNIRS data using a Hitachi ETG-4000 near-infrared spectroscopy device.

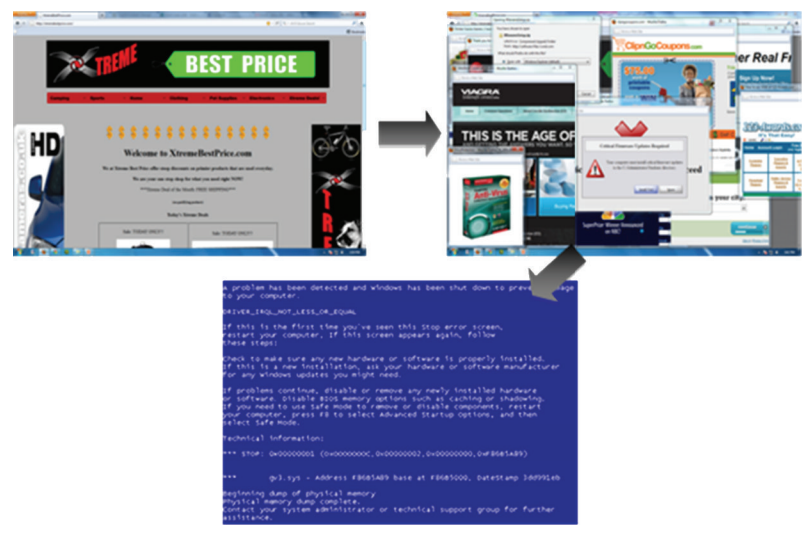

FIGURE 3: Screenshots of the day four manipulations. Figure 3(a) shows the homepage of xtremebestprice.com, 3(b) shows the popups that occur on the site, and 3(c) shows the "blue screen of death".

Participants wore a cap with 52 channels that take measurements two times per second. As fNIRS equipment is somewhat sensitive to movement, participants were placed at a comfortable distance from the keyboard and mouse and were asked to minimize movement throughout the experiment. Prior research has shown that this minimal movement does not corrupt the fNIRS signal with motion artifacts [53]. All fNIRS data were synchronized by placing marks in the datasets whenever a task started or ended, or whenever a manipulation occurred. We collected GSR data using a wireless Affectiva Q-Sensor bracelet. We examined the participants' electrodermal activity (EDA) with the GSR sensor.

2.7. Survey Instruments. At the end of each 15-minute session, participants filled out postsession surveys that included the NASA Task Load index (TLX) for subjective workload assessment, a semantic differential survey [1], and the SelfAssessment Manikin (SAM) for valence and arousal emotional state assessment. Lang's SAM [2] has been used to identify a number of emotional states that fall on the 2dimensional valence-arousal schema developed by Russell. Semantic differential surveys measure the connotative meaning of concepts. Participants were asked: "Place a mark on each scale to indicate your feelings or your opinions regarding the time you spent today working on the computer and browsing through the various websites searching for bikes." They then had to indicate how they felt during that day's tasks by placing a mark on a scale defined by two bipolar adjectives (for example, "Adequate-Inadequate," "EnjoyableFrustrating," or "Difficult to use-Easy to use"). Embedded within a list of adjective pairings was the adjective pairing of "Trusting-Distrusting". We included this pairing within the longer list to subjectively gauge levels of trust in a way that would not cause users to become suspicious of our research paradigm.

2.7.1. End of Experiment Survey. As described previously, after the final experiment session and the subject debriefing, 
we asked the subjects to complete a final postsurvey that asked them three questions (did they notice a slow-down on day three?; if so, to what did they attribute the slow-down?; during day four, what were their thoughts about the causes of the computer issues?).

2.8. Measures of Valence and Arousal. Lang's Self-Assessment Manikin (SAM) survey has been used by many researchers to acquire subjects' self-report valence and arousal. From an objective, real-time measurement point of view, the galvanic skin response (GSR) sensor is also capable of measuring arousal. GSR sensors measure changes in electrical resistance across two regions of the skin, and the electrical resistance of the skin fluctuates quickly during mental, physical, and emotional arousal. This change in the skin's electrodermal activity (EDA) can be used to measure arousal in individuals, although not valence. Despite this limitation, GSR has been used in controlled experiments to measure arousal while participants experienced a variety of emotions such as stress, excitement, boredom, and anger $[27,54]$.

2.9. Manipulation Checks. We checked our manipulations by reviewing participant responses to the postexperiment openended survey and by analyzing the subject results on the postsession surveys. All participants reported that they believed the malware they encountered (the malware manipulation) was the result of their visit to a malevolent website, which they believe that they had stumbled across on their own via their Google searches. The slow internet manipulation introduced on day three received mixed responses from participants. Five participants noted feeling suspicious of the researchers during the third experiment session - they suspected that we were behind the slow internet manipulation. We examine the data from this subset of subjects at the end of this paper.

\section{Results}

Survey, GSR, and fNIRS data were recorded for all 11 participants. Figure 4 provides a graph of these trends, averaged across subjects, over the course of days two, three, and four.

We aimed to determine whether or not there were statistically significant differences between the baseline, slowed internet manipulation, and malware manipulation sessions. We made statistical comparisons of our survey, GSR, and fNIRS datasets. All $t$-test results are available in Table 1 and they are discussed in the analysis section of this paper. Paired comparison $t$-tests were conducted on our postsession survey responses.

The GSR data were analyzed using paired sample onetailed $t$-tests to compare the electrodermal activity (EDA) values before and after target manipulations. On day three, we compared EDA immediately before the onset of the slowed internet manipulation and then 100 seconds after the slowed internet manipulation began. GSR data for two subjects were discarded due to poor contact with the skin on the third measurement day and substantial motion artifacts in the data. On day four, we used a paired sample one-tailed $t$-test to compare the EDA values immediately before the onset of

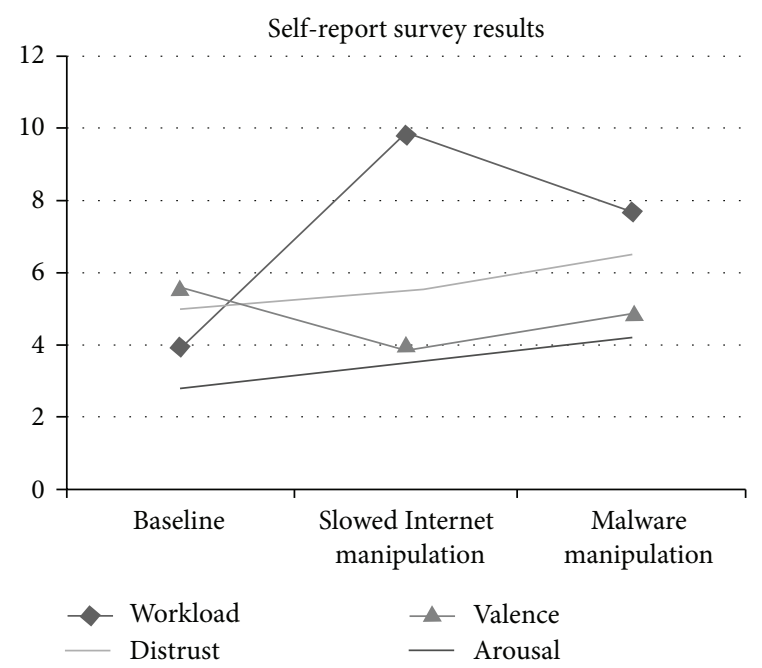

FIGURE 4: Self-report values after the baseline, slowed internet manipulation, and malware manipulation.

the faux computer virus manipulation and then immediately after the 'Blue Screen of Death' appeared for each subject. Data for three subjects were discarded due to poor contact with the skin on the fourth measurement day and substantial motion artifacts in the data. Therefore, results were computed for the remaining eight subjects' data.

We used the NIRS_SPM MATLAB suite of tools to analyze the fNIRS data [55]. We first converted our raw light intensity data into relative changes of oxygenated $(\mathrm{HbO})$ concentrations. We then preprocessed all data using a bandpass filter (between 0.1 and $0.01 \mathrm{~Hz}$ ) to remove noise and motion artifacts. We used a general linear model (GLM) to fit our fNIRS data. Because the GLM analysis relies on the temporal variational pattern of signals, it is robust to differential path length factor variation, optical scattering, or poor contact on the head. By incorporating the GLM with a $P$-value calculation, NIRS-SPM not only enables calculation of activation maps of $\mathrm{HbO}$ but also allows for spatial localization. We used Tsuzuki's 3D-digitizer-free method for the virtual registration of NIRS channels onto the stereotactic brain coordinate system. Essentially, this method allows us to place a virtual optode holder on the scalp by registering optodes and channels onto reference brains. Assuming that the fNIRS probe is reproducibly set across subjects, the virtual registration can yield as accurate spatial estimation as the probabilistic registration method. Please refer to the following paper for further information [56]. Based on Tsuzuki's virtual anatomical registration findings, we identified the functional regions of the brain that were activated during the slowed internet manipulation. The top of Figure 5 shows that areas with significantly higher $\mathrm{HbO}$ were the Frontopolar area and the Dorsolateral prefrontal cortex (DLPFC). For day four, the bottom of Figure 5 shows the area of the brain where $\mathrm{HbO}$ significantly increased when subjects transitioned from the control time (searching for bikes with no manipulations) to the virus manipulation (i.e., high arousal and, presumably, alarm). 
TABLE 1: $P$ values for $t$-tests comparing the effects of the manipulations on the dependent variables.

\begin{tabular}{|c|c|c|c|c|c|c|}
\hline & Distrust $(\uparrow)$ & Workload $(\uparrow)$ & Valence $(\downarrow)$ & Arousal $(\uparrow)$ & GSR-EDA $(\uparrow)$ & fNIRS HbO $(\uparrow)$ \\
\hline $\begin{array}{l}\text { Comparison between baseline and } \\
\text { the slowed Internet manipulation } \\
\text { sessions }\end{array}$ & 0.095 & $0.001^{*}$ & $0.003^{*}$ & $0.018^{*}$ & $0.002^{*}$ & $\begin{array}{l}\text { DLPFC, frontopolar } \\
\text { region }\end{array}$ \\
\hline \multirow[t]{2}{*}{$\begin{array}{l}\text { Comparison between baseline and } \\
\text { the malware manipulation sessions }\end{array}$} & $0.006^{*}$ & $0.004^{*}$ & 0.120 & $0.003^{*}$ & $0.49^{*}$ & $\begin{array}{l}\text { DLPFC, orbitofrontal } \\
\text { cortex, broca's area, } \\
\text { frontopolar region }\end{array}$ \\
\hline & Distrust $(\uparrow)$ & Workload $(\downarrow)$ & Valence $(\uparrow)$ & Arousal $(\uparrow)$ & GSR-EDA $(\uparrow)$ & fNIRS $\mathrm{HbO}^{*}$ \\
\hline $\begin{array}{l}\text { Comparison between slowed } \\
\text { Internet manipulation and malware } \\
\text { manipulation sessions }\end{array}$ & 0.084 & 0.074 & 0.105 & $0.035^{*}$ & 0.19 & N/A \\
\hline
\end{tabular}

${ }^{*}$ indicates statistical significance. Note that $n=11$ for all comparisons, except the GSR data, which is noted above.

The brain regions that showed significant activation during the malware manipulation were the frontopolar area, DLPFC, orbitofrontal area, and the pars triangularis Broca's area. Figure 5 shows the results of our statistical analysis on the fNIRS data transposed onto a standard brain. These results are also noted in an abbreviated form in Table 1.

\section{Interpretation and Analysis of Hypotheses}

Our first set of hypotheses stated the following.

H1a. If unexpected computer-generated stimuli are negative, but minor, they will cause reactions similar to being frustrated and/or annoyed. More specifically, when compared to a user's baseline state, unexpected minor negative stimuli will be associated with a moderate increase in arousal and a large decrease in valence, as indicated by the letter "H1a" in Figure 2.

$H 1 b$. If unexpected computer-generated stimuli are perceived as severely negative, or they are attributed to possible malintent by an external agent, they will cause individuals to feel afraid and/or alarmed. More specifically, when compared to a user's baseline state, the presence of these unexpected and severely negative stimuli will be associated with a large increase in arousal and only a moderate decrease in valence, as indicated by the letter "H1b" in Figure 2.

$\mathrm{H} 1$ Corollary. The regions identified by $\mathrm{Hla}$ and $\mathrm{H} 1 \mathrm{~b}$ in Figure 2 can be distinguished noninvasively, with fNIRS and/or GSR sensors.

The survey data shows an increase in frustration and arousal and a decrease in valence. Furthermore, the survey data suggests that a loss in trust was correlated with these emotional changes (although the change was not statistically significant; see Table 1). Figure 6 shows the average valence and arousal reports for days two, three, and four overlaid onto Russell's circumplex model. The slow Internet and malware manipulations had their expected effect on participants, with the self-report valence and arousal scores for the slow internet manipulation residing in the region of $\mathrm{Hla}$ and the malware manipulation residing in the region of $\mathrm{H} 1 \mathrm{~b}$.

The GSR data are consistent with the arousal survey data, indicating that subjects' level of arousal increased during the frustrating, slowed internet manipulation. This increase in arousal while frustrated also appears in the previously noted research on arousal and emotion. Furthermore, the fNIRS data shows that this frustrating manipulation was accompanied by an increase in DLPFC and frontopolar activation. The DLPFC is involved in working memory, emotion regulation, and self-control [57]. The frontopolar and DLPFC findings are consistent with a wealth of neuroscience research that ties together negative affect (such as frustration) with a need to conduct emotion regulation and with an increase in cognitive load.

The malware manipulation was designed to elicit alarm and lower trust. The survey data shows that a significant increase in self-report frustration and arousal, and a decrease in self-report valence, are associated with the malware manipulation. The GSR data are consistent with the arousal survey data, indicating that subjects' level of arousal increased during the alarmful malware manipulation. Additionally, survey data suggests that a statistically significant loss in trust was reported after the malware manipulation (again, see Table 1), indicating that trust was correlated with these emotional state changes.

The fNIRS data shows that the malware manipulation was accompanied by an increase in brain activation [45] in DLPFC, pars triangulate Broca's area, and the orbitofrontal cortex. Researchers believe that the pars triangulate Broca's area is responsible for helping humans to turn subjective experiences into speech. Research has found that high levels of arousal (presumably related to alarm if valence is negative) have a direct effect on Broca's area [41]. It is possible that the alarm experienced by our users caused an increase in activation of Broca's area while they attempted to find words to comprehend what was occurring. All 11 subjects did use the "Call Researcher" button after they saw the "Blue Screen of Death," and all 11 subjects reported in their postexperiment interview that they truly believed a virus had been placed on their computer by the xtremebestprice.com website. Anecdotally, we noted the difficulty in producing speech during this condition when one subject simply repeated "What?" again and again to himself in a slow manner during the virus manipulation. The DLPFC, as mentioned previously, plays a role in emotion regulation and has been found to be activated during negative affect situations [45]. Lastly, the 

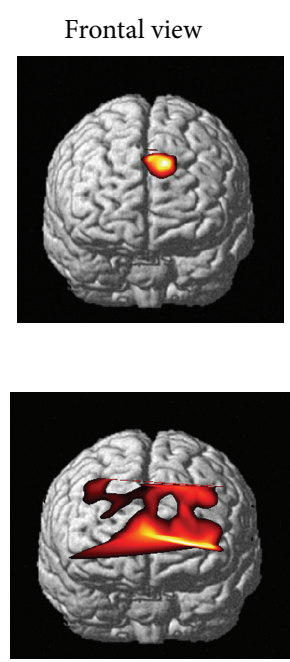

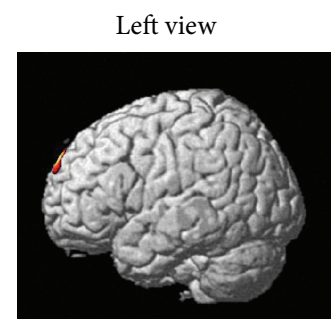

(a)

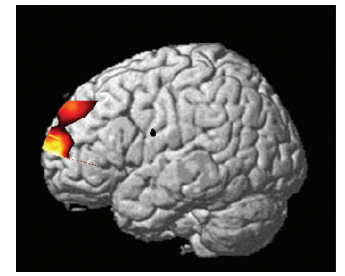

(b)
Right view

No significant region of activation

Figure 5: (a) Significant areas of activation comparing the slow_internet ${ }_{\Delta \mathrm{HbO}}$ to before_slow_internet ${ }_{\Delta \mathrm{HbO}}$. $(n=11)$. (b) Significant areas of activation while subjects encountered the computer virus $(n=11)$.

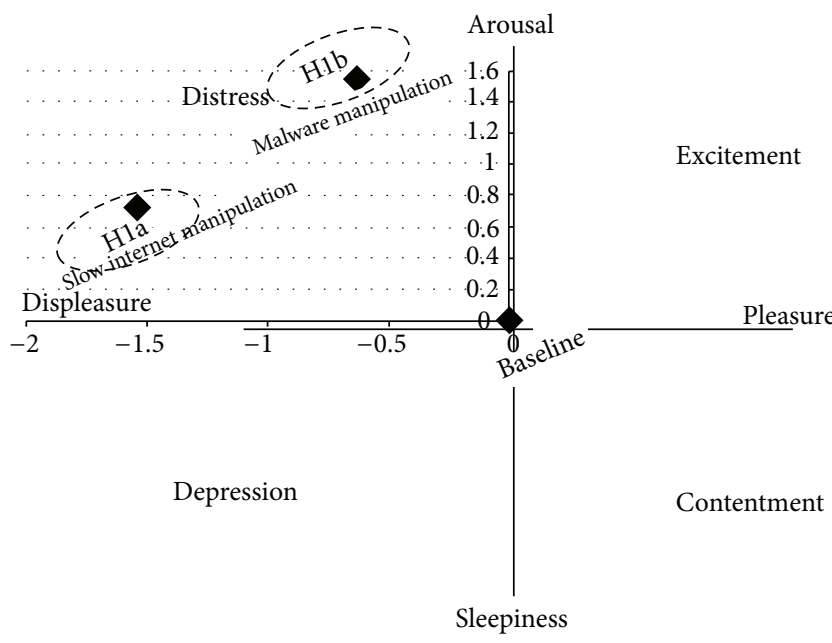

FIGURE 6: Valence and arousal reports for each session are overlaid on Russell's circumplex model. We subtracted the baseline (day two) arousal and valence measures from days two, three, and four to shift the data so that baseline was at point $(0,0)$ in Russell's model.

increase in activation in the orbitofrontal cortex makes sense, as activation in that region has been linked to high stress situations $[42,45,58]$.

We also note that there was an increase in EDA during both manipulations (as compared to baseline), but unfortunately the GSR data was not able to significantly differentiate between the day four (malware) and day three (slow Internet) user states. However, the fNIRS was able to distinguish between the user states in these two conditions. The slow Internet manipulation was associated with increased DLPFC and frontopolar activation, while the malware manipulation was associated with increased activation in Broca's area, the DLPFC, the frontopolar region, and the orbitofrontal cortex.
The activation in these specific brain regions was somewhat expected, as prior research (described in the literature review section) has tied these regions to emotional states such as alarm, frustration, and stress.

Our first set of hypotheses were thus supported by our results, affirming the notion that it is feasible to not only (1) identify the emotional effects of the computer manipulations, but also (2) distinguish between minor and major negative stimuli via subjects' differential emotional reactions. And, by using the fNIRS device, this distinction can be made relatively noninvasively, while users work on their computer system under normal working conditions.

Our second hypothesis stated that computer users will experience more cognitive load when interacting with a malfunctioning computer than they did when working on a properly functioning machine.

Our postsurvey results and our fNIRS results support this hypothesis. The NASA-TLX self-report workload scores showed a significant increase after the slowed internet manipulation, which was also associated with a reduction in trust (see Table 1). The NASA-TLX scores also increased significantly after subjects' trust was lowered by the malware manipulation. Furthermore, the fNIRS data during the slowed Internet manipulation and during the malware manipulation showed that the frontopolar region, an area associated with cognitive load, was significantly higher during each of these manipulations than compared to baseline levels. The frontopolar region is involved in much of the higher order cognitive processing that makes us human, such as executive processing, memory, and planning. Although the elusiveness of this region makes it difficult to determine specific functionality, we can safely assume that the increased $\mathrm{HbO}$ in this region during the slowed internet manipulation indicates an overall increase in cognitive load. To repeat, our second hypothesis was supported by our surveys and by the fNIRS device. 
TABle 2: $P$ values for $t$-tests comparing the effects of the day 2 versus day 3 manipulations on the dependent variables in the study.

\begin{tabular}{|c|c|c|c|c|c|c|c|}
\hline \multirow{2}{*}{ Groups } & \multicolumn{5}{|c|}{ Self-report measures } & \multicolumn{2}{|r|}{ Sensor measures } \\
\hline & Distrust $(\uparrow)$ & Workload $(\uparrow)$ & Frustration $(\uparrow)$ & Valence $(\downarrow)$ & Arousal $(\uparrow)$ & GSR-EDA $(\uparrow)$ & fNIRS HbO $(\uparrow)$ \\
\hline Not suspicious & $0.038^{*}$ & $0.029^{*}$ & $0.007^{*}$ & $0.026^{*}$ & 0.104 & 0.15 & Middle temporal gyrus*, DLPFC* \\
\hline Suspicious & no change & $0.003^{*}$ & $0.008^{*}$ & $0.038^{*}$ & $0.035^{*}$ & $0.012^{*}$ & Paracingulate cortex ${ }^{*}$ \\
\hline
\end{tabular}

${ }^{*}$ indicates statistical significance.

4.1. Exploratory Analysis of Suspicion. Hypotheses 3 and $3 a$ were hypotheses about the concept of suspicion.

H3. Increases in paracingulate cortex activity will be associated with increased suspicion in individuals as they conduct the cognitively demanding process involved in inferring the intentions of others.

$H 3 a$. Suspicion will be accompanied by an increase in physiological indices of arousal.

Given the dearth of research on the construct of suspicion, we conducted some exploratory analyses on this concept. After subjects were debriefed about the true nature of the study we asked subjects whether or not they had become suspicious that the researchers were actually responsible for the manipulations. All subjects reported that they believed the malware manipulation on the fourth day, was truly a computer virus that they had stumbled across. Interestingly, five subjects reported that they felt suspicious of the experimenters during the slowed Internet manipulation on the third day. One subject described his/her reaction as: "I felt a little suspicious that the experimenters were messing around with the computer, but I kept telling myself I was just being paranoid." Thus, we post hoc split our sample into subjects who, at the end of day 3, mentioned "suspicion" of the experimenters or other agents $\left(n_{1}=5\right)$ and those who did not $\left(n_{2}=6\right)$. Note that these are small sample sizes; more research is needed to further validate these results.

Regarding Hypothesis 3 (suspicion will be associated with increases in paracingulate cortex activity), the participants who interacted with the frustrating slowed Internet manipulation and reported no associated suspicion had significant activation in their DLPFC and their middle temporal gyrus. As noted before, the DLPFC activation suggests an increase in emotion regulation and cognitive load during this condition. The middle temporal gyrus subserves language and semantic memory processing and is connected, through a series of networks, to the frontopolar region. This cognitive load is likely directly related to the semantic processing needed to complete the Internet browsing task. Thus, the fNIRS indicates that the nonsuspicious subjects simply became frustrated by the manipulation, and their brain activity showed this increase in cognitive load and the need for emotion regulation that is associated with frustration. In contrast, for the suspicious subjects, the area of activation was directly above the anterior cingulate cortex (ACC), which is the frontal part of the cingulate cortex that resembles a "collar" form around the corpus callosum. The paracingulate cortex is a subset of the ACC. It is located within the ACC, closest to the external brain cortex.
Thus, our fNIRS results lend support to our third hypothesis. We also note that our subject pool was small, and the post hoc splitting of groups into suspicious and nonsuspicious was based on exit interviews. We thus consider these findings tentative, although intriguing.

We also computed summary data for the suspicious and nonsuspicious groups separately (see Table 2). We looked at the self-report scores reported after the day two (baseline trust) session and after the day three (slowed Internet manipulation) session. We compared the GSR EDA measures for each group immediately before the slowed manipulation began, and then 100 seconds after the slow internet manipulation began.

All empirical trends were as expected. That is, the slowed internet manipulation increased cognitive load, frustration, and arousal and decreased valence for both subgroups. However, only the suspicious group showed a statistically significant increase in arousal during the slow Internet manipulation (both in terms of self-report and GSR measures), thus supporting Hypothesis $3 \mathrm{a}$.

Lastly, it is worth noting that the construct of trust may also be correlated with the emotional and cognitive changes described above. As described previously, one item in the postsession survey asked subjects to indicate their level of trust while working through the experiment that day. High trust was listed on the left side of the scale (high trust $=1$ ) and high distrust was listed on the far right side of the scale (distrust $=7$ ). As we expected, participants lost trust after the third and fourth sessions (after day two, three, and four Likert survey averages were $M=4.9,5.5$, and 6.6, resp.). We computed one-tailed, paired comparison $t$-tests by using day two data as a baseline for our comparisons. As reported in Table 1, participants reported feeling less trusting after the malware manipulation (day four) than they reported after the control, day-two session $(P<0.0065)$. Loss of trust also occurred after the slow Internet manipulation (day three) when compared to the control, day-two session, but this difference was not statistically significant $(P<0.0950)$. These results suggest a correlation between trust and the dependent measures reported in this experiment. Future studies should explore this relationship further, by using a more systematic manipulation of trust and by employing more robust surveys from the trust literature to measure changes in in the different facets of trust.

\section{Conclusion}

One overarching goal in this study was to demonstrate the feasibility of using fNIRS to objectively measure the cognitive 
and emotional correlates of two computer manipulations in real time. In particular, when we slowed down participants' Internet speeds we caused reactions similar to being frustrated and/or annoyed. In this scenario, we hypothesized that, when compared to a user's baseline state, unexpected minor negative stimuli would be associated with a moderate increase in arousal and a large decrease in valence. Our fNIRS, GSR, and survey data supported this hypothesis. When we simulated a computer virus and crash on users' systems, we designed the manipulation to be deemed severely negative, and to be attributed to possible malintent by an external agent. In this scenario, we hypothesized that when compared to a user's baseline state, the presence of these unexpected and severely negative stimuli would be associated with a large increase in arousal and only a moderate decrease in valence. Again, our fNIRS, GSR, and survey data supported this hypothesis. We also looked at suspicion in our datasets in a post hoc manner, with a small subject pool. While we did find support for the claim that the Theory of Mind region of the paracingulate cortex is activated during suspicion, we make those claims with caution. Future work must take a closer look at the state of suspicion. In particular, fNIRS could be used with experimental paradigms that specifically manipulate suspicion in order to get a more reliable measure of that state. The same can be said for the construct of trust. Results from the trust item in our postsession surveys suggested that trust is associated with the emotional and cognitive state changes that we measured, but there is a need to conduct follow-on studies that manipulate trust in a more controlled (e.g., counterbalanced) experimental paradigm.

The results also suggest that fNIRS can measure cognitive activity related to users' changing cognitive and emotional states during human-computer interactions. This is quite promising, as the noninvasive fNIRS device is easy-to-set up and comfortable, and it has been implemented wirelessly, showing great promise for future measurements of computer users' experiences while they work with computer systems in real time. The results also indicate that trust and suspicion are correlated with the cognitive and emotional state changes of the computer users. Future research should attempt to disentangle these findings and to look more specifically at manipulations of trust and suspicion in order to measure those constructs during human-computer interactions.

\section{Conflict of Interests}

The authors declare that there is no conflict of interests regarding to the publication of this paper.

\section{Acknowledgment}

The authors would like to thank the Air Force Office of Sponsored Research (AFOSR) for support of this research.

\section{References}

[1] S. G. Hart and L. E. Staveland, "Development of NASA-TLX (Task Load Index): results of empirical and theorical research," in Human Mental Workload, P. Hancock and N. Meshkati, Eds., pp. 139-1183, 1988.

[2] M. M. Bradley and P. J. Lang, "Measuring emotion: the selfassessment manikin and the semantic differential," Journal of Behavior Therapy and Experimental Psychiatry, vol. 25, no. 1, pp. 49-59, 1994.

[3] E. R. Thompson, "Development and validation of an internationally reliable short-form of the positive and negative affect schedule (PANAS)," Journal of Cross-Cultural Psychology, vol. 38, no. 2, pp. 227-242, 2007.

[4] R. C. Mayer and J. H. Davis, "The effect of the performance appraisal system on trust for management: a field quasiexperiment," Journal of Applied Psychology, vol. 84, no. 1, pp. 123-136, 1999.

[5] B. McEvily and M. Tortotiello, "Measuring trust in organizational research: review and recommendations," Journal of Trust Research, vol. 1, no. 1, pp. 23-63.

[6] P. Sackett and J. Larson, "Research strategies and tactics in industrial and organizational psychology," in Handbook of Industrial and Organizational Psychology, M. D. Dunnette and L. M. Hough, Eds., vol. 1, pp. 419-490, Consulting Psychologists Press, Palo Alto, Calif, USA, 1990.

[7] A. L. Edwards, "The relationship between the judged desirability of a trait and the probability that the trait will be endorsed," Journal of Applied Psychology, vol. 37, no. 2, pp. 90-93, 1953.

[8] D. Grimes, D. S. Tan, S. E. Hudson, P. Shenoy, and R. P. N. Rao, "Feasibility and pragmatics of classifying working memory load with an electroencephalograph," in Proceedings of the 26th Annual CHI Conference on Human Factors in Computing Systems (CHI '08), pp. 835-844, Florence, Italy, April 2008.

[9] A. Gevins and M. Smith, "Neurophysiological measures of cognitive workload during human-computer interaction," Theoretical Issues in Ergonomics Science, vol. 4, no. 1-2, pp. 113-131, 2003.

[10] O. Gruber and D. Y. von Cramon, "The functional neuroanatomy of human working memory revisited: evidence from 3-T fMRI studies using classical domain-specific interference tasks," NeuroImage, vol. 19, no. 3, pp. 797-809, 2003.

[11] L. M. Hirshfield, E. T. Solovey, A. Girouard et al., "Brain measurement for usability testing and adaptive interfaces: an example of uncovering syntactic workload in the brain using functional near infrared spectroscopy," in Proceeding of the 27th annual SIGCHI Conference on Human Factors in Computing Systems, 2009.

[12] L. M. Hirshfield, R. Gulotta, S. Hirshfield et al., "This is your brain on interfaces: enhancing usability testing with functional near-infrared spectroscopy," in Proceedings of the 29th Annual CHI Conference on Human Factors in Computing Systems (CHI '11), pp. 373-382, ACM Press, Vancouver, Canada, May 2011.

[13] A. Etkin, T. Egner, and R. Kalisch, "Emotional processing in anterior cingulate and medial prefrontal cortex," Trends in Cognitive Sciences, vol. 15, no. 2, pp. 85-93, 2011.

[14] G. Hajcak, N. McDonald, and R. F. Simons, "Error-related psychophysiology and negative affect," Brain and Cognition, vol. 56, no. 2, pp. 189-197, 2004.

[15] A. Dimoka, "What does the brain tell us about trust and distrust? Evidence from a functional neuroimaging study," Management Information Systems Quarterly, vol. 34, no. 2, pp. 373-396, 2010.

[16] F. Krueger, K. McCabe, J. Moll et al., "Neural correlates of trust," Proceedings of the National Academy of Sciences of the United States of America, vol. 104, no. 50, pp. 20084-20089, 2007. 
[17] R. Parasuraman and M. Rizzo, Neuroergonomics: The Brain at Work, Oxford University Press, 2008.

[18] L. M. Hirshfield, A. Girouard, E. T. Solovey et al., "Humancomputer interaction and brain measurement using functional near-infrared spectroscopy," in Proceedings of the Symposium on User Interface Software and Technology, ACM Press, 2007.

[19] J. B. Lyons, C. K. Stokes, K. J. Eschleman, G. M. Alarcon, and A. J. Barelka, "Trustworthiness and IT suspicion: an evaluation of the nomological network," Human Factors, vol. 53, no. 3, pp. 219-229, 2011.

[20] C. Wickens, J. Lee, Y. Liu, and S. Becker, An Introduction to Human Factors Engineering, Pearson, 2004.

[21] I.-Y. Son, M. Guhe, W. D. Gray, B. Yazici, and M. J. Schoelles, "Human performance assessment using fNIR," in Biomonitoring for Physiological and Cognitive Performance during Military Operations, vol. 5797 of Proceedings of SPIE, pp. 158-169, Orlando, Fla, USA, April 2005.

[22] D. McBride and D. Schmorrow, Quantifying Human Information Processing, Lexington Books, 2005.

[23] H.-C. Leung, H. Oh, J. Ferri, and Y. Yi, "Load response functions in the human spatial working memory circuit during location memory updating," NeuroImage, vol. 35, no. 1, pp. 368-377, 2007.

[24] A. Gevins, M. E. Smith, L. McEvoy, and D. Yu, "High-resolution EEG mapping of cortical activation related to working memory: effects of task difficulty, type of processing, and practice," Cerebral Cortex, vol. 7, no. 4, pp. 374-385, 1997.

[25] E. E. Smith and J. Jonides, "Storage and executive processes in the frontal lobes," Science, vol. 283, no. 5408, pp. 1657-1661, 1999.

[26] A. Ortony and T. J. Turner, "What's basic about basic emotions?" Psychological Review, vol. 97, no. 3, pp. 315-331, 1990.

[27] R. W. Picard, Affective Computing, MIT Press, Cambridge, UK, 1997.

[28] J. A. Russell, "A circumplex model of affect," Journal of Personality and Social Psychology, vol. 39, no. 6, pp. 1161-1178, 1980.

[29] G. Ball and J. Breese, "Modeling the emotional state of computer users," in Proceedings of the Workshop on Attitude, Personality and Emotions in User-Adapted Interaction, Banff, Canada, 1999.

[30] W. Bosma and E. André, "Exploiting emotions to disambiguate dialogue acts," in Proceedings of the 9th International Conference on Intelligent User Interfaces (IUI '04), pp. 85-92, ACM Press, Madeira, Portugal, January 2004.

[31] D. Rousseau, S. Sitkin, R. Burt, and C. Camerer, "Not so different after all: a cross-discipline view of trust," Academy of Management Review, vol. 23, no. 3, pp. 393-404, 1998.

[32] R. Mayer, J. Davis, and D. Schoorman, "An integrative model of organizational trust," Academy of Management Review, vol. 20, no. 3, pp. 709-734, 1995.

[33] R. J. Lewicki, D. J. McAllister, and R. I. Bies, "Trust and distrust: new relationships and realities," Academy of Management Review, vol. 23, no. 3, pp. 438-458, 1998.

[34] D. McAllister, "Affect- and cognition-based trust as foundations for interpersonal cooperation in organizations," Academy of Management Journal, vol. 38, no. 1, pp. 24-59, 1995.

[35] J. D. Lee and K. A. See, "Trust in automation: designing for appropriate reliance," Human Factors, vol. 46, no. 1, pp. 50-80, 2004.

[36] B. Shneiderman, "Designing trust into online experiences," Communications of the ACM, vol. 43, no. 12, pp. 57-59, 2000.

[37] R. Parasuraman and D. H. Manzey, "Complacency and bias in human use of automation: an attentional integration," Human Factors, vol. 52, no. 3, pp. 381-410, 2010.
[38] P. Bobko, A. Barelka, and L. M. Hirshfield, "The construct of state-level suspicion: a model and research agenda for automated and information technology (IT) contexts," Human Factors, vol. 56, no. 3, pp. 489-4508, 2014.

[39] H. Kober, L. F. Barrett, J. Joseph, E. Bliss-Moreau, K. Lindquist, and T. D. Wager, "Functional grouping and cortical-subcortical interactions in emotion: a meta-analysis of neuroimaging studies," NeuroImage, vol. 42, no. 2, pp. 998-1031, 2008.

[40] T. Colibazzi, J. Posner, Z. Wang et al., "Neural systems subserving valence and arousal during the experience of induced emotions," Emotion, vol. 10, no. 3, pp. 377-389, 2010.

[41] A. M. Hull, "Neuroimaging findings in post-traumatic stress disorder," British Journal of Psychiatry, vol. 181, pp. 102-110, 2002.

[42] C. Hooker and R. Knight, "The role of lateral orbitofrontal cortex in the inhibitory control of emotion," in The Orbitofrontal Cortex, D. H. Zald and S. Rauch, Eds., p. 307, 2006.

[43] B. Shneiderman and C. Plaisant, Designing the User Interface: Strategies for Effective Human-Computer Interaction, AddisonWesley, Reading, Mass, USA, 4th edition, 2005.

[44] R. Parasuraman and D. Caggiano, Neural and Genetic Assays of Human Mental Workload, in Quantifying Human Information Processing, Lexington Books, 2005.

[45] A. Golkar, T. B. Lonsdorf, A. Olsson et al., "Distinct contributions of the dorsolateral prefrontal and orbitofrontal cortex during emotion regulation," PLoS ONE, vol. 7, no. 11, Article ID e48107, 2012.

[46] D. Premack and G. Woodruff, "Does the chimpanzee have a theory of mind?" Behavioral and Brain Sciences, vol. 1, no. 4, pp. 515-526, 1978.

[47] J. Stiff, K. Hyun, and R. Closepet, "Truth biases and aroused suspicion in relational deception," Communication Research, vol. 19, no. 3, pp. 326-345, 1992.

[48] J. K. Burgoon, J. P. Blair, and R. E. Strom, "Cognitive biases and nonverbal cue availability in detecting deception," Human Communication Research, vol. 34, no. 4, pp. 572-599, 2008.

[49] B. Chance, Z. Zhuang, U. Chu, C. Alter, and L. Lipton, "Cognition activated low frequency modulation of light absorption in human brain," Proceedings of the National Academy of Sciences of the United States of America, vol. 90, no. 8, pp. 3770-3774, 1993.

[50] K. Izzetoglu, S. Bunce, M. Izzetoglu, B. Onaral, and K. Pourrezaei, "fNIR spectroscopy as a measure of cognitive task load," in Proceedings of the 25th Annual International Conference of the IEEE Engineering in Medicine and Biology Society, pp. 34313434, Cancun, Mexico, September 2003.

[51] A. Girouard, E. Solovey, L. M. Hirshfield et al., "Distinguishing difficulty levels with non-invasive brain activity measurements," in Proceedings of the 12th IFIP TC 13 International Conference on Human-Computer Interaction, pp. 440-452, Uppsala, Sweden, 2009.

[52] L. M. Hirshfield, K. Chauncey, R. Gulotta et al., "Combining electroencephalograph and near infrared spectroscopy to explore users' mental workload states," in Proceedings of the 5th International Conference on Human-Computer Interaction, pp. 239-247, Springer, San Diego, Calif, USA, 2009.

[53] E. T. Solovey, A. Girouard, K. Chauncey et al., "Using fNIRS brain sensing in realistic hci settings: experiments and guidelines," in Proceedings of the 22nd Annual ACM Symposium on User Interface Software and Technology (UIST '09), pp. 157-166, ACM Press, Victoria, Canada, October 2009. 
[54] M.-Z. Poh, N. C. Swenson, and R. W. Picard, "A wearable sensor for unobtrusive, long-term assessment of electrodermal activity," IEEE Transactions on Biomedical Engineering, vol. 57, no. 5, pp. 1243-1252, 2010.

[55] J. C. Ye, S. Tak, K. E. Jang, J. Jung, and J. Jang, "NIRS-SPM: statistical parametric mapping for near-infrared spectroscopy," NeuroImage, vol. 44, no. 2, pp. 428-447, 2009.

[56] D. Tsuzuki, V. Jurcak, A. K. Singh, M. Okamoto, E. Watanabe, and I. Dan, "Virtual spatial registration of stand-alone fNIRS data to MNI space," NeuroImage, vol. 34, no. 4, pp. 1506-1518, 2007.

[57] E. Berkman and M. Lieberman, "Using neuroscience to broaden emotion regulation: theoretical and methodological considerations," Social and Personality Psychology Compass, vol. 3, no. 4, pp. 475-493, 2009.

[58] J. L. Hanson, M. K. Chung, B. B. Avants et al., "Early stress is associated with alterations in the orbitofrontal cortex: a tensor-based morphometry investigation of brain structure and behavioral risk," The Journal of Neuroscience, vol. 30, no. 22, pp. 7466-7472, 2010. 

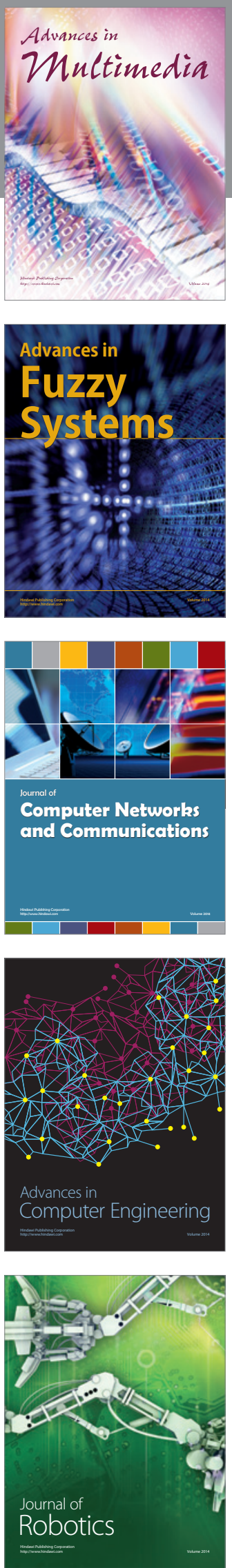

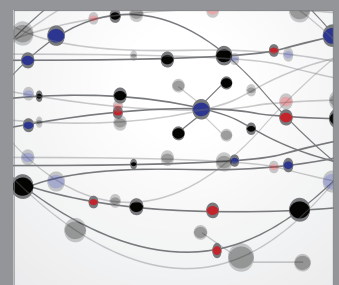

The Scientific World Journal
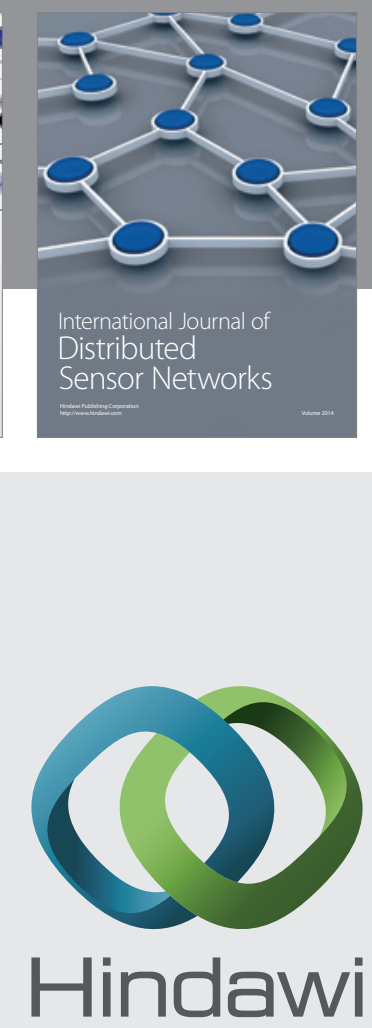

Submit your manuscripts at

http://www.hindawi.com
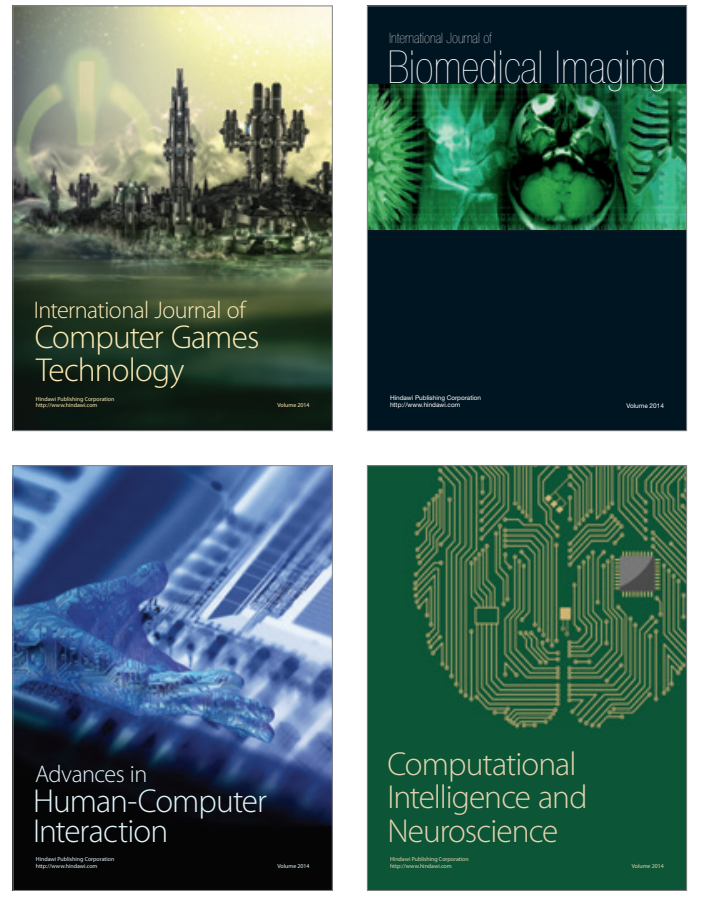
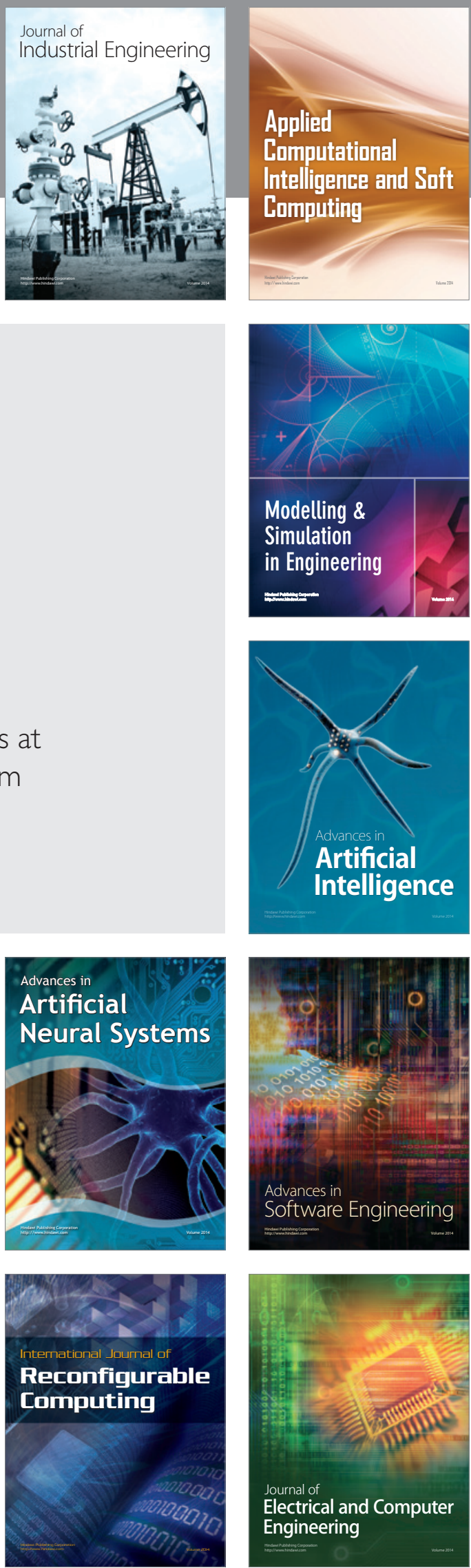